\title{
Multiphase flow and cavitation - Comparison of Flow in Rectangular and Circular Nozzle
}

\author{
Jana Jablonská ${ }^{1, a}$, and Marian Bojko ${ }^{1}$ \\ ${ }^{1}$ Department of Hydromechanics and Hydraulic Equipment, Faculty of Mechanical Engineering, VŠB-Technical \\ University of Ostrava, 17. listopadu 15, 70833 Ostrava, Czech republic
}

\begin{abstract}
The paper describes the experimental measurement of cavitation domain at multiphase turbulent flows in rectangular and circular nozzle. The flowing medium is water. Both nozzles were measured at maximum flow range without saturation of circuit by air (without cavitation and with cavitation). Subsequently, the article describes the changes in the sizes of cavitation domain during multiphase flow if the circuit is saturated by the known amount of air. In the article the sizes of cavitation domain with and without air saturating are presented for both nozzles. Subsequently the frequency of cavitation vortices are also calculated using Strouhal number. These measured values and the effect of air content in the circuit will be further used for subsequent numerical modelling and the results will be verified each other.
\end{abstract}

\section{Cavitation phenomenon}

Cavitation is a phenomenon which is currently widely discussed and is the subject of many publications, not only as an issue but also as a beneficial effect. Efforts to intensify energy transfer exist, and as a consequence, hydraulic systems, valves and hydraulic mechanism elements are operated in conditions of slight cavitation, due to which the components suffer from cavitation wear. This ultimately reduces the service life of the equipment.

Cavitation is characterised by creating, developing, and decay of bubbles when a certain state of the liquid at the given site is attained. This state is determined by the pressure and temperature of the liquid. Actually, the liquid flow continuity is disturbed. The cavitation bubbles are entrained by the flowing liquid and carried away to a higher pressure area, where they decay sharply (i.e. implode). If this effect occurs near to or on the wall bypassed by the liquid, the wall material is stressed and eroded appreciably. Cavitation bubbles forming part of the flow are referred to as the cavitation region. A cavitation region is highly unstable: cavitation bubbles change the sites of their creation and decay. Therefore, stability of the cavitation region shape is monitored in space and time. Cavitation bubbles appear at sites where the strength of the liquid is disturbed. The highest degrading effect on the strength of the liquid is exerted by free undissolved gases, constituting cavitation nuclei. Hence, absolutely pure water has a sufficient strength and no cavitation bubbles are formed in it.

\footnotetext{
${ }^{\mathrm{a}}$ Corresponding author: jana.jablonska@vsb.cz
}

If the liquid is in a saturated state, it becomes supersaturated on pressure reduction, and vapours of the liquid are evolved. This phenomenon is called by vapour cavitation. Alternatively, if the liquid pressure in the bubble (cavitation nucleus) area is lowered slowly, the bubble may grow appreciably due to dissolved gas diffusion from the surroundings into the bubble. This phenomenon is called by air (gaseous) cavitation. Diffusion of gas accompanying vapor cavitation is very slow and it can often be neglected. Both of these variants occur simultaneously and are referred to as gas cavitation. If the liquid contains bubbles with large amounts of air, i.e. with large initial radii, then pressure reduction is accompanied by constant bubble growth without evaporation. This effect is called pseudocavitation.

The most frequent cause of cavitation in hydraulic machines is dynamic pressure decrease when the liquid passes through channels or when it bypasses barriers; this phenomenon is called hydrodynamic cavitation. A vortex, or a vortex shadow, is formed when the liquid flow bypasses a barrier, the frequency of the whirls so formed being determined by the Strouhal number

$$
S h=\frac{f \cdot d}{v}
$$

where $f$ is the frequency of the vortices, $d$ is the height of the protrusions, and $v$ is the velocity of the free stream flow. $[1,6]$

This suggests that the cavitation region also exhibits periodicity. According to [9], a cavitation cloud formed in a diffuser is periodic by nature, and the Strouhal 
number is lying within the range of $(0.31 ; 0.58)$ depending on the diffuser type defined by Eq. (1),

$$
S h=\frac{f \cdot l_{k}}{v}
$$

where $l_{k}$ is the cavitation region length.

Cavitation regions are classed $[1,2]$ in dependence on shape as:

- Pocket regions - a cluster of cavitation bubbles fills completely a certain space; this occurs, e.g., in nozzles, on blades of hydraulic machines, and the like

- Fibrous regions (gap cavitation) - the cavitation bubbles form a visible sequence in the form of fibres; this occurs, e.g., at the ends of water turbine blades or of ship propeller blades

- Rectangular regions - forming on bypassed surfaces, e.g. on blades of pumps, turbines and propellers.

Actually, gas cavitation (by evolution of gas or evaporation of the liquid) and pseudo cavitation are two inseparable phenomena. Both are present together, although one or the other may prevail. [1]

Cavitation is governed by the saturated vapour pressure, which is temperature dependent and can be found in the literature. Evolution of dissolved air depends on the time during which the liquid is subjected to reduced pressure. For longer nozzles, more air is released and cavitation takes place at a higher pressure. The pressure required for the start of cavitation with respect to the air content of the liquid obeys the empirically found dependence $[1,2]$.

Cavitation noise, arising during the growth of the cavitation bubbles and their pulsations, is typical manifestation of cavitation. It is particularly during the decay of a bubble that the pressure and velocity in the bubble's surroundings attain high levels and propagate spherically away from the source. Since a large number of such bubbles are present in the cavitation region, the pressure waves from the bubbles affect one another. The summary effects of all the bubbles result in cavitation noise or vibrations. Cavitation noise of the individual bubbles fall largely the ultrasonic frequency range (up to $3 \mathrm{MHz}$ ). Large pulsating cavitation bubbles in welldeveloped cavitation, however, have a low frequency (down to $10 \mathrm{~Hz}$ ), which is related to the periodic nature of the cavitation regions arising due to the bypassing of barriers. [5]

As the demands put on hydraulic systems are increasing, cavitation starts to occur not only in systems with water but also in oil devices. Since the physical properties of water and oils differ, existing insight into cavitation in water can be only partly applied to oil.

The scope of this issue is very broad and so a narrower area of scientific interest must be specified. This publication will be primarily focussed on experimental investigation of cavitation in nozzles for water. The results will be used as boundary conditions for mathematical modelling of cavitation with respect to the air content and for verification of mathematical models of cavitation. Subsequently, the experiment will be used to verify the mathematical model especially of pseudo cavitation. In the next stage, the experience gained will be transposed to flowing oil.

\section{Experimental methods}

Cavitation is investigated in two ways: experimentally and mathematically. Experimental studies confirm that cavitating flow depends significantly on the geometry and on the flow conditions. The methods are as follows:

- Visualisation, which is a method using video camera recording; a tomographic method which is based on the capacitance or resistance principle, which will allow the circular cavitation regions to be examined both in water and in oil; a bubble spectrometer investigates the bubble distribution in dependence on the radius, including the distribution density, optical methods

- Pressure and noise measurement

The following approaches can be applied to mathematical modelling of liquid flow with cavitation:

- Single phase flow modelling, which is easier as regards the speed of calculation and the mathematical model (Matlab SimHydraulic and Ansys - Fluent) but provides only crude information on the cavitation regions

- Multiphase flow modelling (Fluent) for mixtures of a liquid, vapour and any additional undissolved compressible gases with cavitation (Ansys - Fluent). The boundary conditions, particularly the amount of undissolved air in the liquid, must be specified for this approach. The start and development of gas cavitation and pseudo cavitation and their mutual effect can then be investigated. [3]

\section{Experimental equipment}

The experimental equipment consists of a hydrogenerator connected to a tank with water and driven by a motor with revolution regulation serving to control the flow rate by frequency converter. A system to saturate the circuit with air is included downstream of the hydro generator. A nozzle serving to generate cavitation follows. The circuit includes pressure sensors and a flowmeter. Water is used as the working liquid. [4]

The scope of the measurement was limited by the equipment design and material parameters.

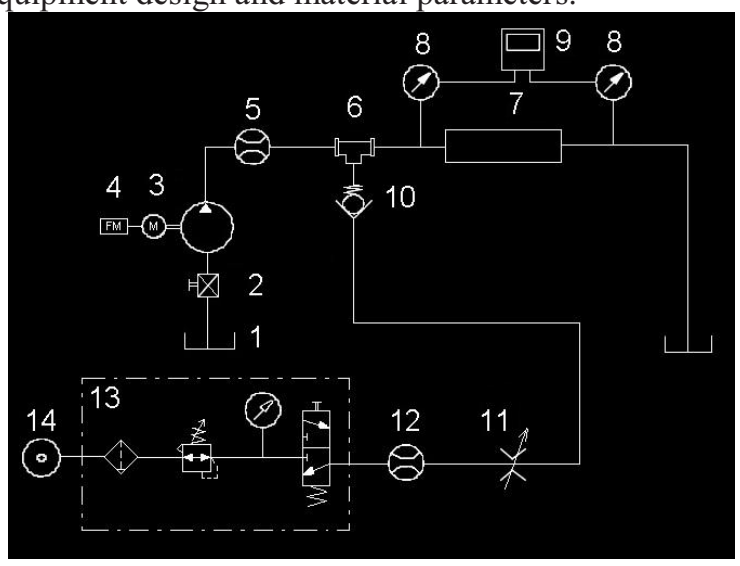

Fig. 1. Circuit scheme - water and air segments 
Circuit description - figure 1, figure 2, figure 3 :

1 - tank, 2 - ball valve, 3 - hydro-generator GRUNDFOS s.r.o. with a motor (type CR 20-5A-F-A-E HQQE), 4 - frequency converter type YASKAWA VS mini J7, 5 - induction flowmeter ELIS PLZEN a.s. (type DN50 PN16 TG ISO 110), 6 - saturation with air, 7 object measured (rectangular or circular nozzle), 8 pressure sensor, 9 - evaluation equipment Hydrotechnik, 10 - one-way valve, 11 - throttle valve to control air flow rate, 12 - air flow meter, 13 - pressure regulating valve with a filter unit and a condensate separator, 14 compressed air source with a pressure gauge.

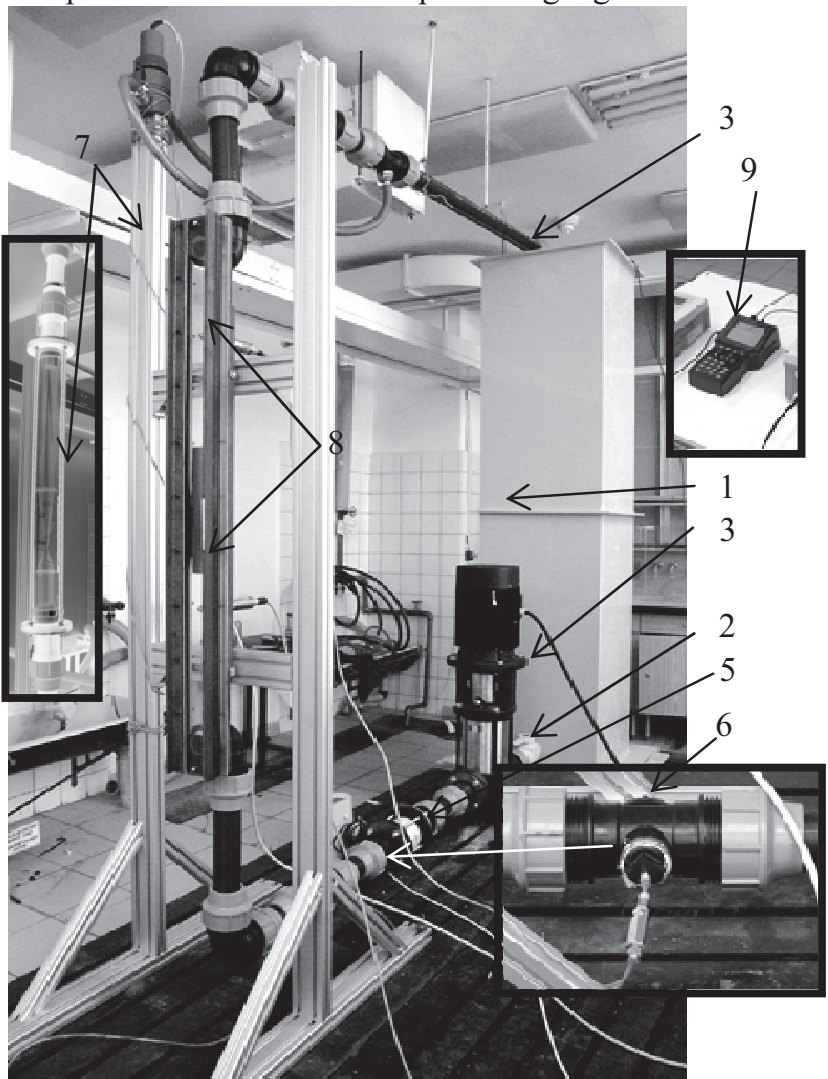

Fig. 2. Photograph of the circuit - water segment

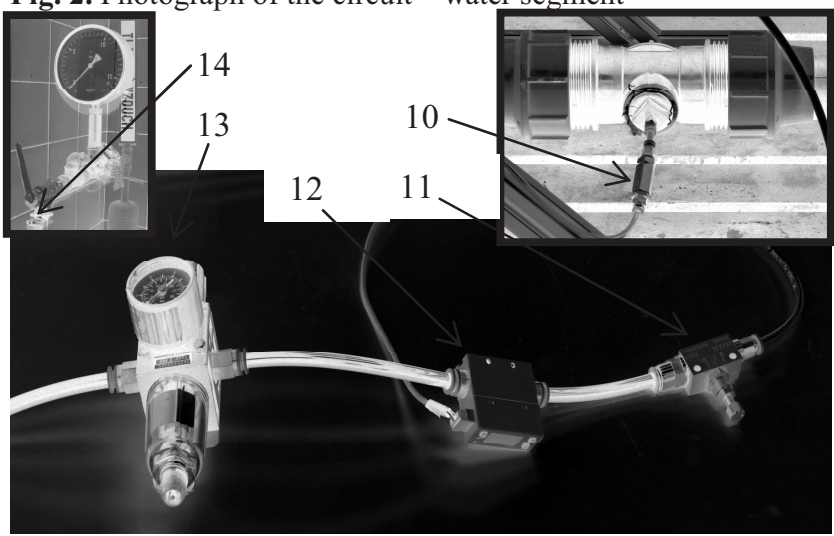

Fig. 3. Photograph of the circuit - air segment
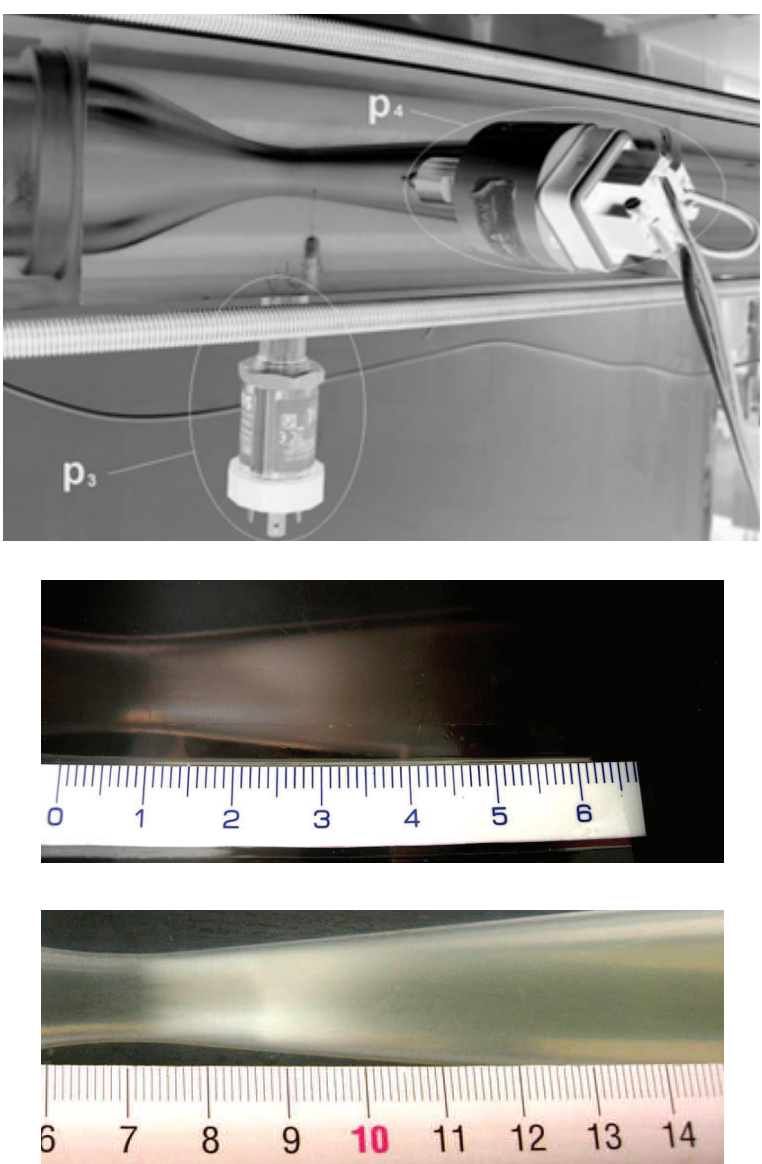

Fig. 4. Nozzle - sensor connection in the narrowest point and comparison of the cavitation region with sensors and without sensors

The nozzles of circular cross section and of rectangular cross section were used for the measurement. Both nozzles are based on the same curve geometry, which is indicatively described in figure. 5 The used material is transparent plastic.

Figure 5 shows that the extension of the diffuser is less than the recommended one at which no flow separation regions are observed. The maximum angle of extension is $12^{\circ}$ (see [1]). In used geometry this angle is $7.3^{\circ}$.

The nozzle of circular cross section was more difficult to production due to turning towards the narrowest place and required high geometrical accuracy. Because the entire area of the nozzle is made from one piece, it is possible to say clearly, that there can be no air sucking at its narrowest place, where underpressure is observed. Due to uncontrolled air sucking in the narrowest nozzle area there is not possible to put any measuring instrument at this place. The nozzle of rectangular cross section was produced by milling a groove (shapes see figure 5) into panel. Subsequently seal of a given shape is placed around milled groove and covered with another plate - a sandwich configuration. Every parts of nozzle are connected with the help of the screws. In the upper panel (see figure 2, figure 9) the holes for connecting the nozzle to the circuit are drilled. This connection is horizontal (both nozzles are positioned vertically) and the straight part before the nozzle is sufficiently long to stabilize of velocity profile. After the first test the seal was ticked to 
the transparent plastic and screws were tightened subsequently. Measurements did not confirm the air sucking at the narrowest nozzle place during the cavitation.

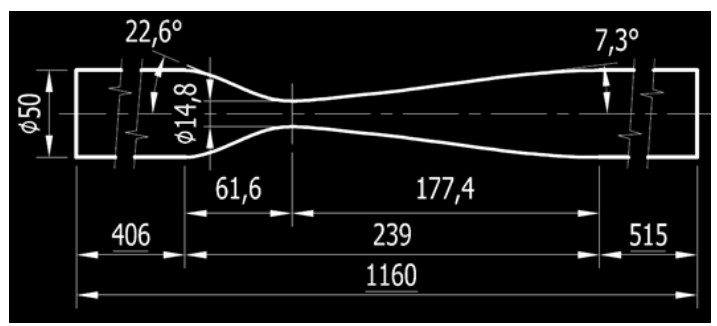

Fig. 5. Rectangular or circular nozzle - curve geometry

\section{The measuring procedure}

The measurement was performed on the circular nozzle. The water flow through the circuit was controlled by means of the frequency converter and the data were recorded with the induction flowmeter. In parallel, pressures upstream, downstream and in the middle of the nozzle were measured.

First, the measurement was performed on the circular nozzle. The water flow through the circuit was controlled by means of the frequency converter and the data were recorded with the induction flow meter. The results indicate that the cavitation region is affected by the connection of the sensor located in the narrowest point of the nozzle (see figure 4), which brought about aspiration of air. For this reason, the cavitation region was evaluated without that pressure sensor (see figure 6).

\section{Evaluation of the experiment}

\section{Experimental results for circular nozzle without air saturating}

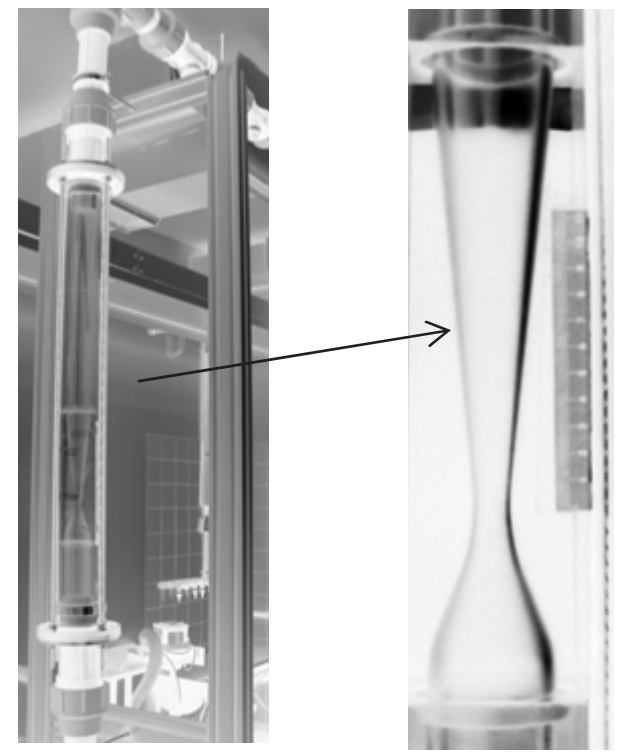

Fig. 6. Circular nozzle

The experiment was performed on the circular nozzle in the first stage (see figure 6). The development of the cavitation region, characterized by the gas layer and noise, was examined for different flow rates (see table 1). By way of illustration, a series of pictures is reproduced (figure 7) showing the growing cavitation region in dependence on the flow rate. It is not clear from figure 7 if the cavitation region is present only at the wall or if it fills (completely or partly) the whole space.

Table 1. Values measured for the circular nozzle

\begin{tabular}{|c|c|c|c|c|c|}
\hline$f$ & $Q_{v}$ & $Q_{v}$ & $L$ & $v_{p}$ & $v_{d}$ \\
\hline$(\mathrm{Hz})$ & $\left(1 . \mathrm{s}^{-1}\right)$ & $\left(\mathrm{m}^{3} \cdot \mathrm{s}^{-1}\right)$ & $(\mathrm{mm})$ & $\left(\mathrm{m} . \mathrm{s}^{-1}\right)$ & $\left(\mathrm{m} \cdot \mathrm{s}^{-1}\right)$ \\
\hline 14 & 2.5 & 0.0025 & 5 & 1.273 & 12.434 \\
\hline 18 & 2.8 & 0.0028 & 20 & 1.426 & 13.926 \\
\hline 22 & 3.1 & 0.0031 & 35 & 1.579 & 15.418 \\
\hline 26 & 3.4 & 0.0034 & 70 & 1.732 & 16.910 \\
\hline 30 & 3.9 & 0.0039 & 100 & 1.986 & 19.397 \\
\hline
\end{tabular}

Table 1 includes values measured for the circular nozzle: $f$ is the frequency set on the frequency converter and matching the water flow rate, $Q_{v}$ is the water flow rate through the system, $L$ is the cavitation region length at the given flow rate, $v_{p}$ is flow velocity in the piping and $v_{d}$ is flow velocity in the narrowest point of the nozzle (i.e. the maximum average velocity).

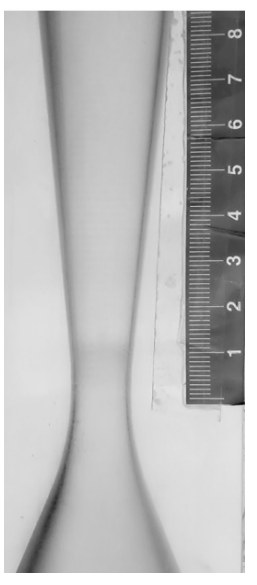

$14 \mathrm{~Hz}$

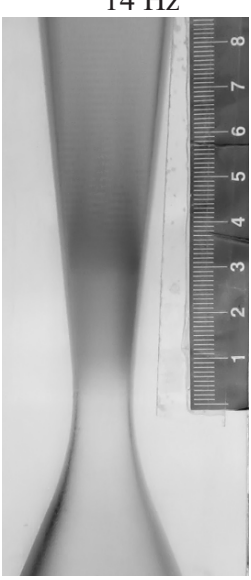

$26 \mathrm{~Hz}$

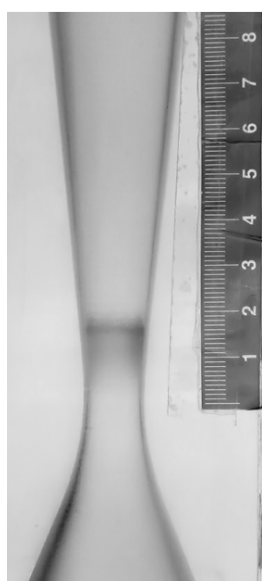

$18 \mathrm{~Hz}$

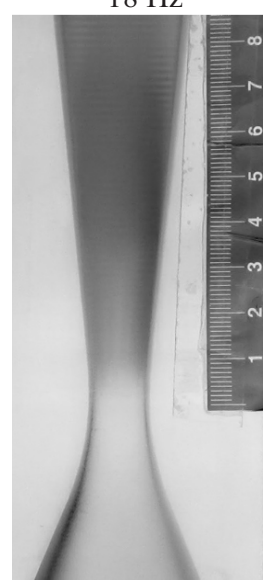

$30 \mathrm{~Hz}$

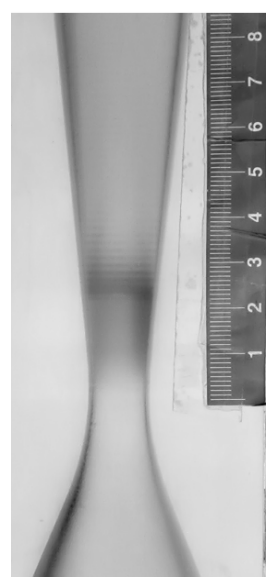

$22 \mathrm{~Hz}$
Fig. 7. Development of the cavitation region in the circular nozzle: measurement without air saturating

Strouhal number for the diffuser is defined by equation (2). Because the exact value of the frequency is 
not being known, the restrictions of Strouhal number can be assumed by [9] in interval $(0.31 ; 0.58)$ for diffuser. The expected range of vortex frequencies can be determined using this limitation. The values of the length of the cavitation field $l_{k}$ and flow velocity are obtained from measurements. During the measurement, it was confirmed for both nozzles, that the vapor region does not change its size significantly, but the gas region with increasing flow rate is increased. In table 2 the estimated vortex frequency values are evaluated for the selected flow rates, which are defined by the frequency set on the drive pumps $f_{\text {pump. }}$. Evaluation is made for measurement without air saturation. According to currently carrying out research the vortex frequency values are found in a given range by table 2 .

Table 2 Strouhal number for a nozzle of circular cross section, without air saturating

\begin{tabular}{|c|c|c|c|c|c|c|c|c|c|}
\hline $\mathrm{f}_{\text {pump }}$ & $Q_{v}$ & $1_{\mathrm{k}}$ & \multicolumn{7}{|c|}{$\mathrm{St}(-)$} \\
\hline$(\mathrm{Hz})$ & $\left(1 . \mathrm{s}^{-1}\right)$ & $(\mathrm{m})$ & 0.31 & 0.35 & 0.4 & 0.45 & 0.5 & 0.55 & 0.58 \\
\hline 16 & 2.6 & 0.01 & 41.0 & 46.3 & 53.0 & 59.6 & 66.2 & 72.8 & 76.8 \\
\hline 18 & 2.8 & 0.02 & 22.1 & 25.0 & 28.5 & 32.1 & 35.7 & 39.2 & 41.4 \\
\hline 22 & 3.1 & 0.035 & 14.0 & 15.8 & 18.0 & 20.3 & 22.6 & 24.8 & 26.2 \\
\hline 26 & 3.4 & 0.07 & 7.7 & 8.7 & 9.9 & 11.1 & 12.4 & 13.6 & 14.3 \\
\hline 30 & 3.9 & 0.1 & 6.2 & 7.0 & 7.9 & 8.9 & 9.9 & 10.9 & 11.5 \\
\hline
\end{tabular}

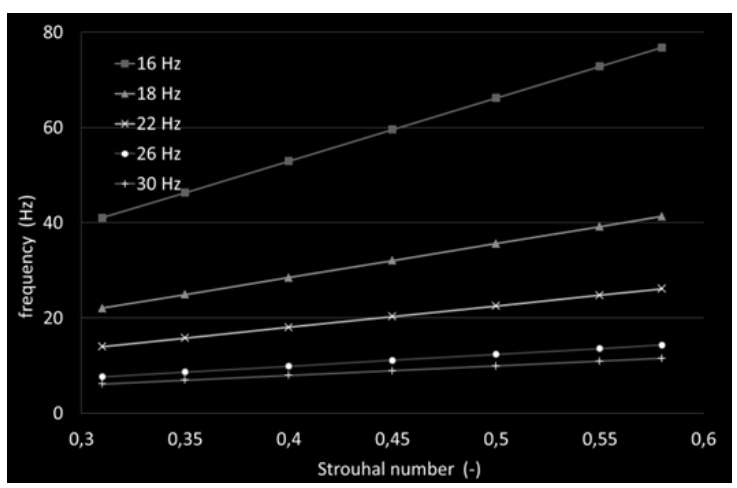

Fig. 8. Vortex frequency versus Strouhal number (different flow rate)

The graph in figure 8 shows, that the vortex frequency decreases with increasing flow rate (frequency of the pump).

\section{Experimental results for circular nozzle with air saturating}

This experiment was repeated in the same conditions, applying air saturation. A series of measurements at $18 \mathrm{~Hz}$ and at selected air flow rates is shown in figure 9. The pictures demonstrate that the vapour cavitation region is not changed significantly, the air content in the next nozzle segment is apparent.

Table 3 includes values measured for the circular nozzle: $Q_{v}$ is water flow rate through the system; $Q_{v g}$ is flow rate of air delivered to the system, $\alpha$ is the volume fraction of air delivered to the system, $L$ is the cavitation region length for the given water and air flow rates.

Table 3. Values measured for the circular nozzle at $18 \mathrm{~Hz}$ and various air flow rates

\begin{tabular}{|c|c|c|c|c|}
\hline$Q_{v}$ & $Q_{v g}$ & $Q_{v g}$ & $\alpha$ & $L$ \\
\hline$\left(1 . \mathrm{s}^{-1}\right)$ & $\left(\mathrm{ml}_{\mathrm{N}} \cdot \mathrm{min}^{-1}\right)$ & $\left(1 . \mathrm{s}^{-1}\right)$ & $(\%)$ & $(\mathrm{mm})$ \\
\hline 2.8 & 50 & $3.02 \cdot 10^{-4}$ & 0.011 & 20 \\
\hline 2.8 & 100 & $6.04 \cdot 10^{-4}$ & 0.022 & 21 \\
\hline 2.8 & 150 & $9.05 \cdot 10^{-4}$ & 0.032 & 22 \\
\hline 2.8 & 200 & $1.21 \cdot 10^{-3}$ & 0.043 & 25 \\
\hline 2.8 & 250 & $1.51 \cdot 10^{-3}$ & 0.054 & 26 \\
\hline 2.8 & 300 & $1.81 \cdot 10^{-3}$ & 0.065 & 27 \\
\hline 2.8 & 350 & $2.11 \cdot 10^{-3}$ & 0.075 & 28 \\
\hline 2.8 & 400 & $2.41 \cdot 10^{-3}$ & 0.086 & 29 \\
\hline
\end{tabular}

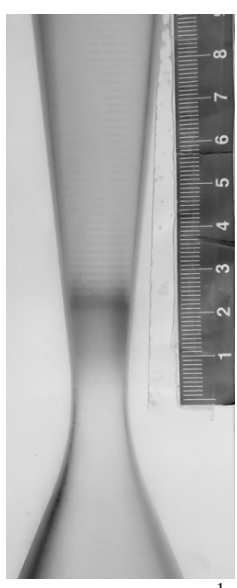

$50 \mathrm{ml}_{\mathrm{N}} \cdot \mathrm{min}^{-1}$

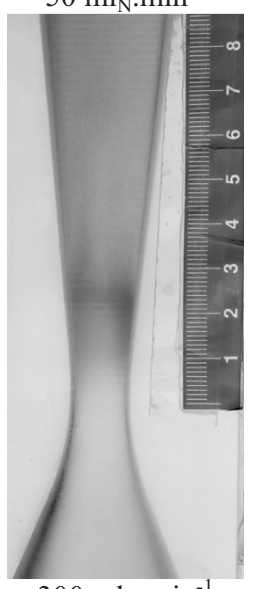

$300 \mathrm{ml}_{\mathrm{N}} \cdot \min ^{-1}$

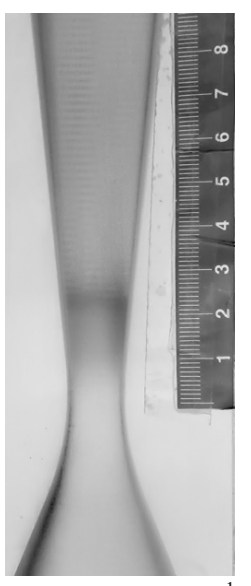

$100 \mathrm{ml}_{\mathrm{N}} \cdot \mathrm{min}^{-1}$

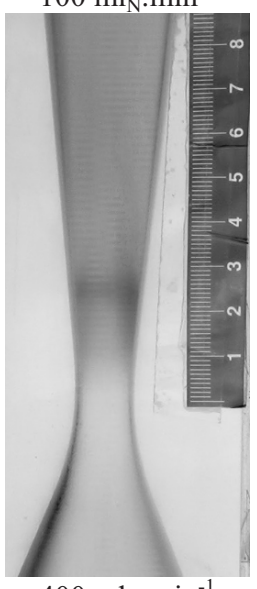

$400 \mathrm{ml}_{\mathrm{N}} \cdot \mathrm{min}^{-1}$

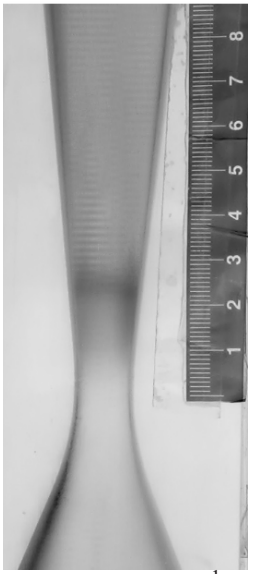

$200 \mathrm{ml}_{\mathrm{N}} \cdot \min ^{-1}$
Fig. 9. Development of the cavitation region in the circular nozzle: measurement at $18 \mathrm{~Hz}$ for various air flow rates $Q_{v z}$

The same theory for evaluation of vortex frequency was used for case of the flow during the circular nozzle with air saturating. Figure 10 describes the dependence of frequency vs. the Strouhal number for uniform flow rate $\left(f_{\text {pump }}=18 \mathrm{~Hz}\right.$ and flow rate $\left.Q_{v}=2.81 \mathrm{~s}^{-1}\right)$ and different air saturating flow rates. It is evident, that with increasing air flow rate the vortex frequencies are decreased. 


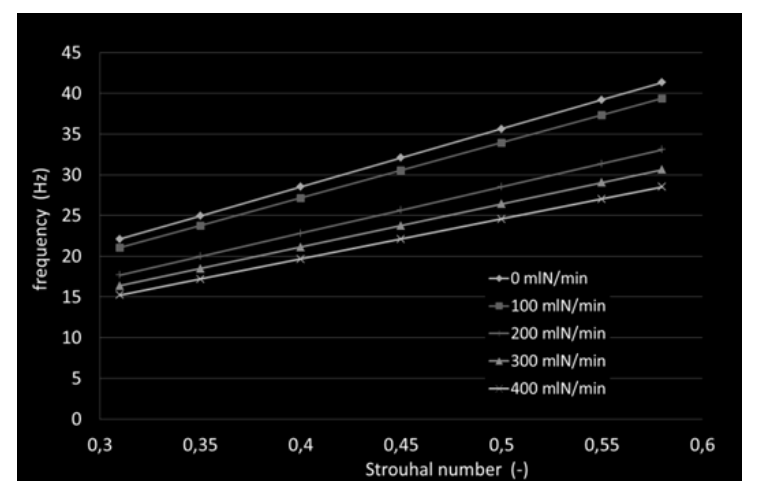

Fig. 10. Vortex frequency versus Strouhal number (different air saturating flow rates)

\section{Experimental results for rectangular nozzle without air saturating}

Since information on the vapour cavitation region location and on air generation in circular nozzle was not so clear, a rectangular nozzle, simpler with respect to the visualization of cavitation region, was designed (see figure 11).
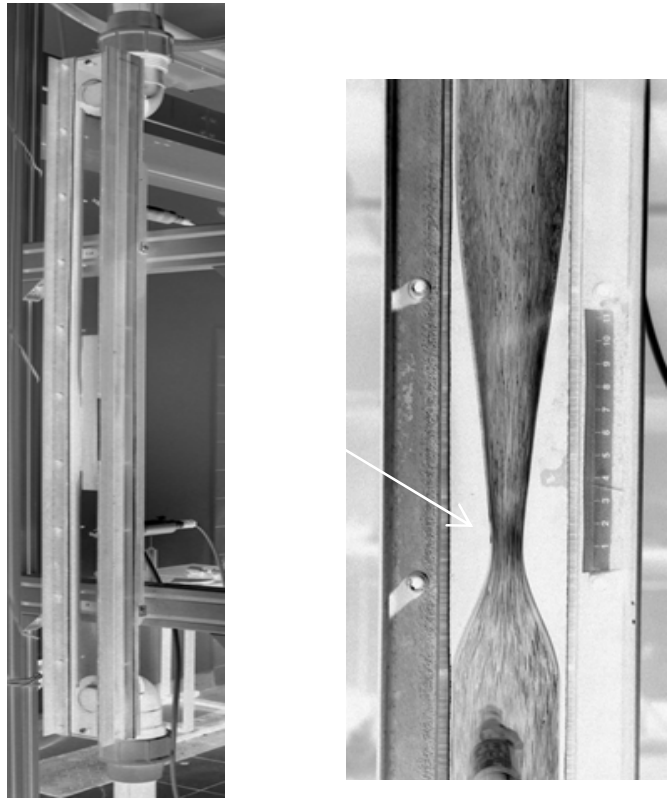

Fig. 11. Rectangular nozzle and detail

The whole circuit was used in the original condition, only circular nozzle was replaced by a rectangular nozzle. Procedure and measurement methodology remained the same, i.e. an evaluation of the size of the cavitation area depending on the flow rate (speed pump), then depending on the saturation of the air without vapour cavitation. The last variant linked these two phenomena, i.e. as vapour cavitation and pseudo-cavitation.

By way of illustration, a series of pictures is reproduced, showing the growing cavitation region in dependence on the flow rate. Figure 12 demonstrates that the cavitation region is only present at the wall while the remaining volume is filled with water. It is evident, that cavitation region is symmetrical and relatively sharply bounded. An important parameter which is changed with increasing flow rate is the noise.
Table 4. Values measured for the rectangular nozzle

\begin{tabular}{|c|c|c|c|c|c|c|}
\hline$f$ & $Q_{v}$ & $L$ & $v_{p}$ & $v_{d}$ & $p_{r, v s .}$ & $p_{r, v y s .}$ \\
\hline$(\mathrm{Hz})$ & $\left(1 . \mathrm{s}^{-1}\right)$ & $(\mathrm{mm})$ & $\left(\mathrm{m} . \mathrm{s}^{-1}\right)$ & $\left(\mathrm{m} . \mathrm{s}^{-1}\right)$ & $(\mathrm{Pa})$ & $(\mathrm{Pa})$ \\
\hline 20 & 2.7 & 0 & 4.50 & 15.20 & 19600 & 44000 \\
\hline 22 & 3.0 & 20 & 5.00 & 16.89 & 25000 & 52000 \\
\hline 24 & 3.2 & 35 & 5.33 & 18.02 & 30000 & 67500 \\
\hline 26 & 3.4 & 55 & 5.67 & 19.14 & 35000 & 86000 \\
\hline 28 & 3.5 & 70 & 5.83 & 19.71 & 40000 & 107000 \\
\hline 30 & 3.7 & 90 & 6.17 & 20.83 & 45000 & 130000 \\
\hline
\end{tabular}
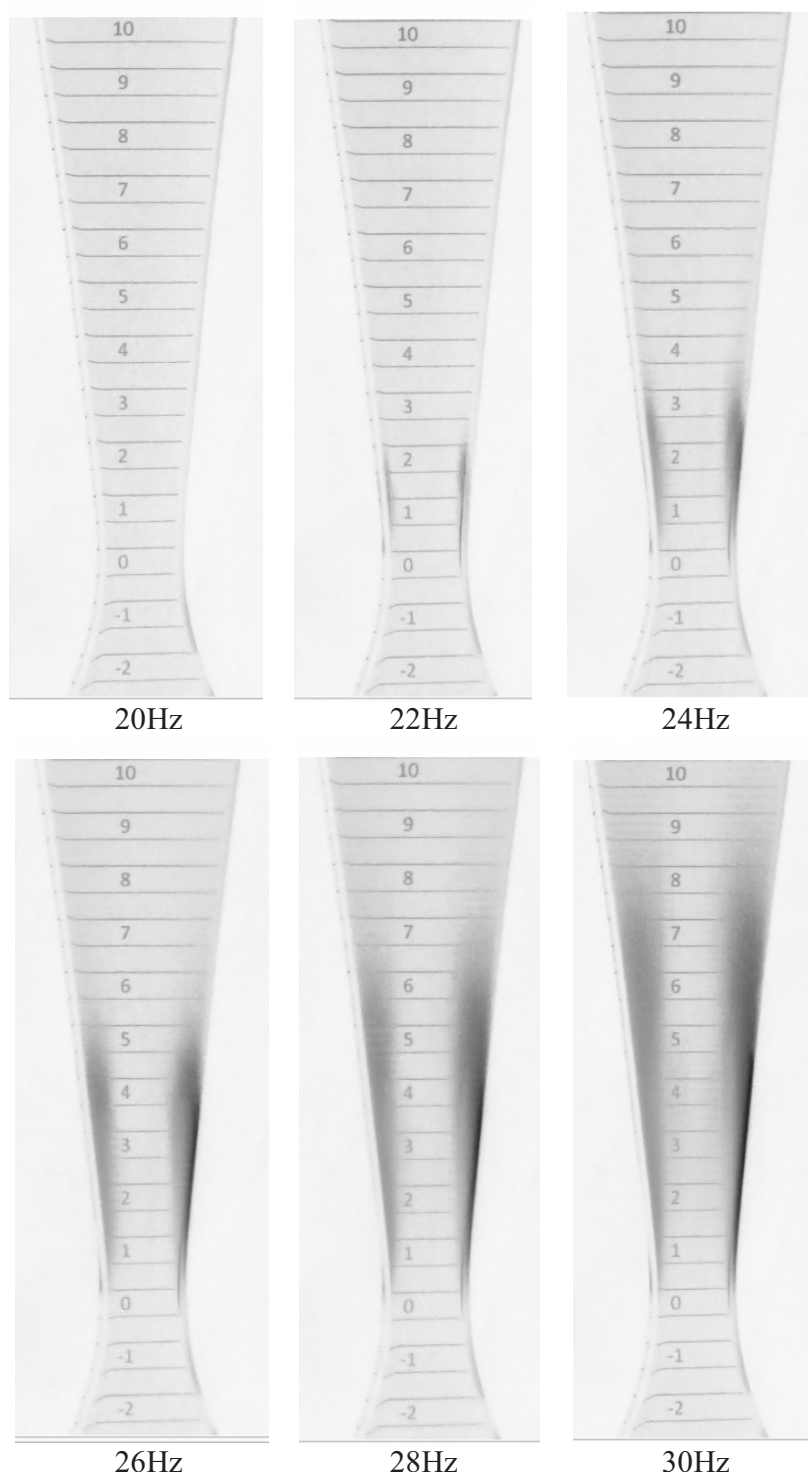

Fig. 12. Development of the cavitation region in the rectangular nozzle: measurement without air saturation at various flow rates

\section{Experimental results for rectangular nozzle with air saturating}

The next step included pseudocavitation testing: the hydrogenerator was set to a speed at which vapour cavitation was not induced. Air was fed to the circuit at various flow rates. The selected measurement variants are shown in figure 13. 
Table 5. Values measured for the rectangular nozzle at $20 \mathrm{~Hz}$ (no cavitation) and various air flow rates

\begin{tabular}{|c|c|c|c|c|}
\hline$Q_{v}$ & $Q_{v g}$ & $Q_{v g}$ & $\alpha$ & $L$ \\
\hline$\left(1 . \mathrm{s}^{-1}\right)$ & $\left(\mathrm{ml}_{\mathrm{N}} \cdot \mathrm{min}^{-1}\right)$ & $\left(1 . \mathrm{s}^{-1}\right)$ & $(\%)$ & $(\mathrm{mm})$ \\
\hline 2.7 & 0 & 0 & 0 & 0 \\
\hline 2.7 & 200 & $1.21 \cdot 10^{-3}$ & 0.045 & 0 \\
\hline 2.7 & 400 & $2.41 \cdot 10^{-3}$ & 0.089 & 0 \\
\hline 2.7 & 600 & $3.62 \cdot 10^{-3}$ & 0.134 & 0 \\
\hline 2,7 & 800 & $4.83 \cdot 10^{-3}$ & 0.179 & 0 \\
\hline 2.7 & 1000 & $6.04 \cdot 10^{-3}$ & 0.223 & 0 \\
\hline
\end{tabular}

The measurements gave evidence that pressure at the inlet and outlet was nearly constant, identical with that in table 5 at the same frequency, i.e. $20 \mathrm{~Hz}$. This is why pressure is not included in table 5 .

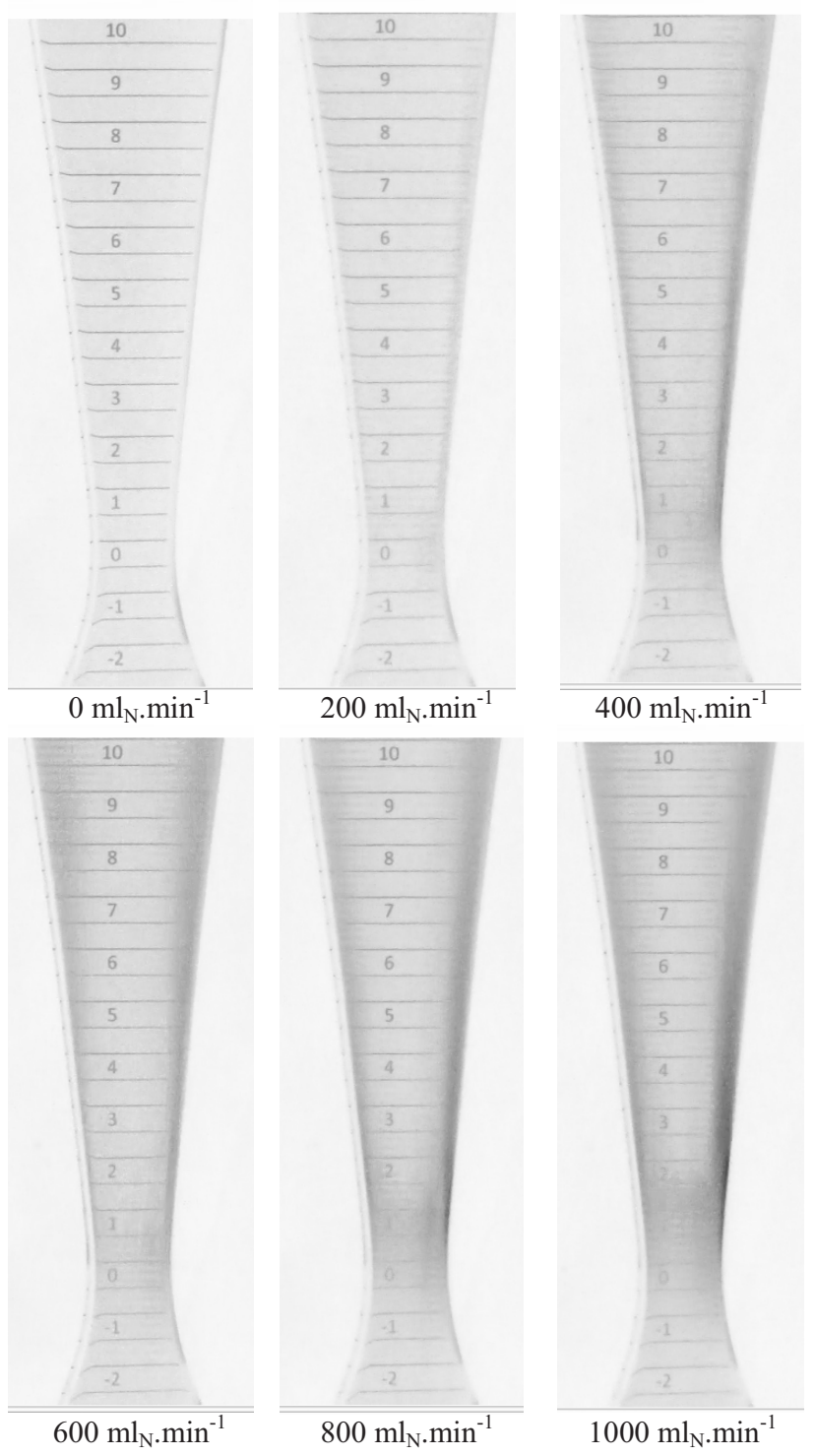

Fig. 13. Development of the cavitation region in the

rectangular nozzle: measurement without cavitation at $20 \mathrm{~Hz}$ and at various air flow rates $Q_{v z}$

Figure 13 demonstrates that the pseudo cavitation region is developed throughout the whole nozzle space, although primarily at the walls. The air region becomes better apparent and noise appears at lower frequencies as the air flow rate is increased. Moreover, air saturation caused pressure increase at the inlet and the pressure reduction at the outlet of the nozzle.

The equipment design permitted the combination of vapour cavitation and pseudocavitation to be evaluated. This can be seen in figure 14 for frequency $26 \mathrm{~Hz}$ (table 6 ) and various air flow rates: vapour cavitation is found to be present at the wall only whereas pseudocavitation fills the whole space in various concentrations.

Table 6. Values measured for the rectangular nozzle at 26 $\mathrm{Hz}$ (with cavitation) and at various air flow rates

\begin{tabular}{|c|c|c|c|c|c|c|}
\hline$Q_{v}$ & $Q_{v g}$ & $Q_{v g}$ & $\alpha$ & $L$ & $p_{r, v s .}$ & $p_{r, v y s .}$ \\
\hline$\left(1 . \mathrm{s}^{-1}\right)$ & $\left(\mathrm{ml}_{\mathrm{N} \cdot} \cdot \mathrm{min}^{-1}\right)$ & $\left(1 . \mathrm{s}^{-1}\right)$ & $(\%)$ & $(\mathrm{mm})$ & $(\mathrm{Pa})$ & $(\mathrm{Pa})$ \\
\hline 3.4 & 100 & $6.04 \cdot 10^{-4}$ & 0,018 & 55 & 89000 & 35000 \\
\hline 3.4 & 200 & $1.21 \cdot 10^{-3}$ & 0,035 & 60 & 89000 & 35000 \\
\hline 3.3 & 300 & $1.81 \cdot 10^{-3}$ & 0,055 & 60 & 90000 & 33000 \\
\hline 3.3 & 400 & $2.41 \cdot 10^{-3}$ & 0,073 & 55 & 92000 & 32000 \\
\hline 3.3 & 500 & $3.02 \cdot 10^{-3}$ & 0,091 & 60 & 93000 & 31000 \\
\hline 3.4 & 600 & $3.62 \cdot 10^{-3}$ & 0,106 & 60 & 94000 & 30000 \\
\hline
\end{tabular}

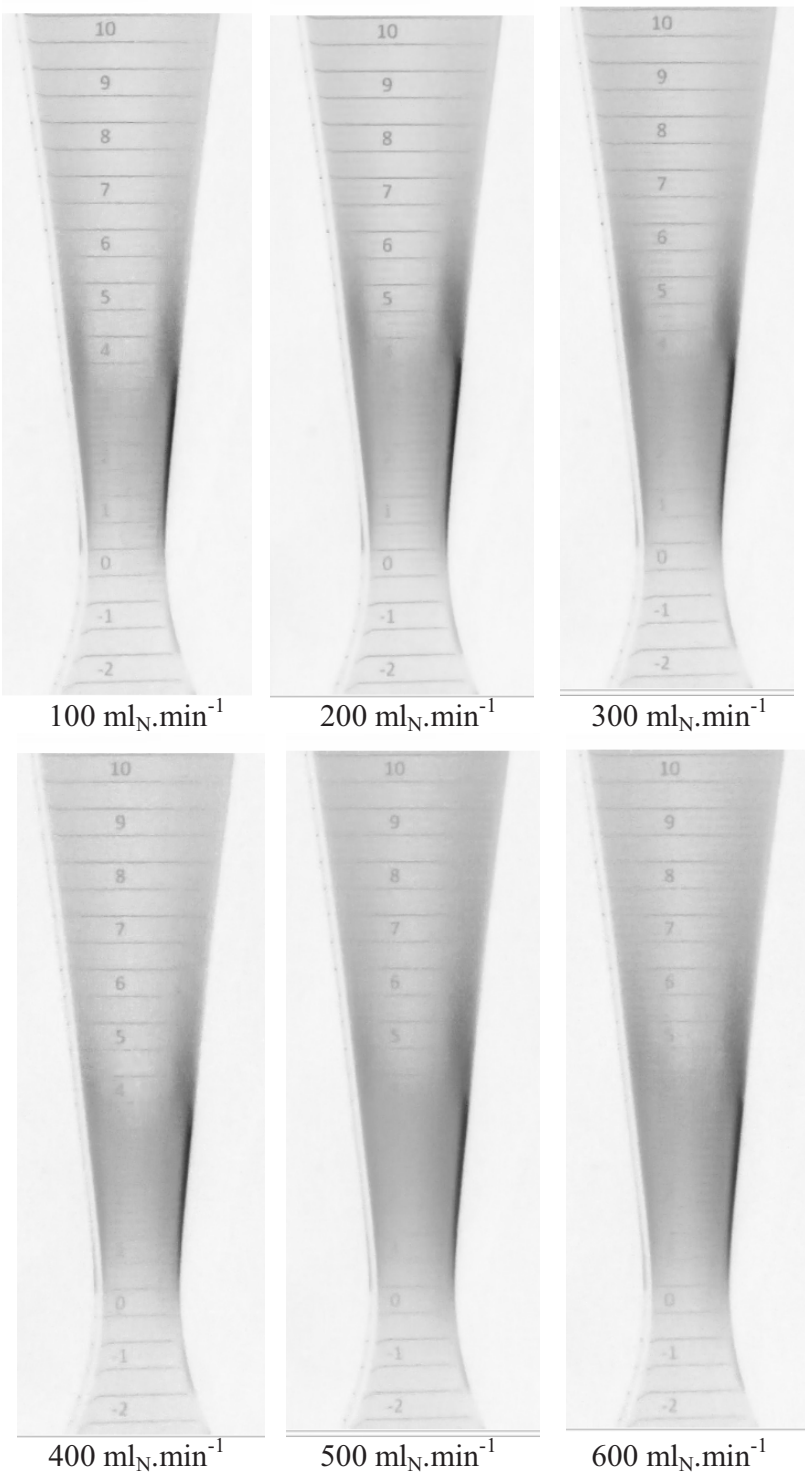

Fig. 14. Development of the cavitation region in the rectangular nozzle: measurement with cavitation at $26 \mathrm{~Hz}$ and at various air flow rates $Q_{v z}$ 


\section{Conclusion}

This work consisted in visual evaluation of the cavitation region in different conditions. The conclusions are as follows.

In the circular nozzle:

- cavitation starts appearing at a flow rate as low as $0.0025 \mathrm{~m}^{3} \cdot \mathrm{s}^{-1}$

- it is impossible to specify whether the cavitation region is present at the wall or within the whole space,

- the amount of vapour released cannot be determined, it can only be inferred from noise during the flow and from the approximate measurements of the cavitation region,

- the size of cavitation region during saturation with air cannot be specified.

In the rectangular nozzle:

- cavitation starts appearing at a higher flow rate, $0.003 \mathrm{~m}^{3} \cdot \mathrm{s}^{-1}$, due to the different geometry

- the cavitation region is present at the wall and is sharply delimited,

- the amount of vapour released cannot be determined, it can only be inferred from noise during the flow,

- the cavitation region during saturation with air is apparent from figure 13 and figure 14; the different cavitation types are assumed to be mutually separated,

- from the noise it can be deduced whether the phenomenon is pseudocavitation or vapour cavitation or a combination of both.

Noise changed as the cavitation region was growing and was different during vapour cavitation and pseudo cavitation. Therefore, in further work also the frequency pulsating cavitation area will be evaluated, as well as the frequency of the measured pressures at the inlet and the outlet of the nozzle and noise.

Vapour cavitation is a problem which is dependent of dissolved air, but the undissolved air plays major role and can be sucked in at a site of reduced pressure, e.g. due to the attachment of a measuring instruments, valves, etc. and will significantly influence the periodicity of the cavitation area.

In the specification and selection of a mathematical model for flow with cavitation it is necessary to know the exact boundary conditions for multiphase flow, otherwise the calculated values of pressures and fluid flow significantly differ from reality. If cavitation region can be monitored through the transparent walls, boundary conditions can be corrected and therefore additionally specified. In the case of opaque walls this correction can be performed using frequency analysis of pressure, noise and vibration measurement. The experiment is therefore used to specify a mathematical model of multiphase flow with vapour and air cavitation, especially boundary conditions and frequency values will be tested further by measurements and especially by mathematical modelling.

\section{Acknowledgements}

This paper has been elaborated in the framework of the project Opportunity for young researchers, reg. no. CZ.1.07/2.3.00/30.0016, supported by Operational Programme Education for Competitiveness and cofinanced by the European Social Fund and the state budget of the Czech Republic.

The work presented in this paper was also supported by a grant SGS "Zkoumání dynamiky hydraulického vedení" SP2014/208

\section{References}

1. Noskievič, J. Kavitace. Praha: Česká akademie věd, 1969. 21-047-69. 280 s. 509-21-875.

2. Noskievič, J. a kol. Kavitace v hydraulických strojích. Praha: SNTL - Nakladatelství technické literatury, 1989. 333 s. ISBN 80-03-00206-0.

3. Kozubková, M. Matematické modely kavitace a hydraulického rázu. Ostrava: VŠB-TU, 2009. 130 s. ISBN 978-80-248-2043-9

4. Jablonská, J. Experimentální zařízení k vzniku a vývoji kavitace v 2D Lavalove dýze. 135/18-12-2013_F. submitted 18. 12. 2013.

5. Himr D. Experimental Fluid Mechanics (EPJ Web of Conferences 2013), 67, No. 02035 (2014)

6. Summers, D. A., Worsey, P. N. The Use Of High Pressure Water Jets To Wash Out Explosives. In: 6th International Conference on Erosion by Liquid and Solid Impact. 1983. p. 5-8.

7. Hružík, M., Vašina, A. Bureček Experimental Fluid Mechanics (EPJ Web of Conferences 2013), 45, No. 01041 (2013)

8. Schubert J. Zařizení pro sycení kapaliny vzduchem. Ostrava, 2011. Bakalářská práce. Fakulta strojní VŠBTU Ostrava

9. Hunsaker J. C. Cavitation Research A Progress Report on Work at the Massachusetts Institute of Technology. Mechanical Engineering (1935) 